\title{
BMJ Open Shared decision-making in advanced kidney disease: a scoping review protocol
}

\author{
Noel Engels (1) , ${ }^{1}$ Gretchen de Graav, ${ }^{2}$ Paul van der Nat, ${ }^{3}$ Marinus van den Dorpel, ${ }^{2}$ \\ Willem Jan Bos, ${ }^{4}$ Anne M Stiggelbout (i) ${ }^{5}$
}

To cite: Engels N, de Graav G, van der Nat P, et al. Shared decision-making in advanced kidney disease: a scoping review protocol. BMJ Open 2020;10:e034142. doi:10.1136/ bmjopen-2019-034142

- Prepublication history and additional material for this paper are available online. To view please visit the journal (http:// dx.doi.org/10.1136/bmjopen2016-34142).

Received 06 September 2019 Revised 31 January 2020 Accepted 03 February 2020
Check for updates

(C) Author(s) (or their employer(s)) 2020. Re-use permitted under CC BY-NC. No commercial re-use. See rights and permissions. Published by BMJ.

${ }^{1}$ Shared decision making, Santeon, Utrecht, Utrecht, The Netherlands

${ }^{2}$ Internal Medicine, Maasstad Ziekenhuis, Rotterdam, ZuidHolland, The Netherlands ${ }^{3}$ Sint Antonius Ziekenhuis, Nieuwegein, Utrecht, The Netherlands

${ }^{4}$ Internal Medicine, Leiden University Medical Center, Leiden, Zuid-Holland, The Netherlands

${ }^{5}$ Medical Decision Making, Leiden University Medical Center, Leiden, Zuid Holland, The Netherlands

Correspondence to

Noel Engels;

n.engels@santeon.nl

\section{ABSTRACT}

Introduction Patients with advanced kidney disease (AKD) have to make difficult treatment modality decisions as their disease progresses towards end-stage kidney disease. International guidelines in nephrology suggest shared decision-making (SDM) to help patients make timely treatment modality decisions that align with their values and preferences. However, systematic reviews or scoping reviews on these SDM interventions and on their reported use or outcomes are lacking. This limits the adoption of SDM in clinical practice and hampers further research and development on the subject. Our aim is to provide a comprehensive and up-to-date overview of these SDM interventions by means of a scoping review of the literature. Scoping reviews can provide a broad overview of a topic, identify gaps in the research knowledge base and report on the types of evidence that address and inform practices. This paper presents our study protocol. Methods and analysis The proposed scoping review will be performed in accordance with the Joanna Briggs Institute's (JBI) methodology for scoping reviews. It will cover both qualitative and quantitative scientific literature, as well as the grey literature on SDM interventions for treatment modality decisions in AKD. Only literature written in English will be considered for inclusion. Two independent reviewers will participate in an iterative process of screening the literature, paper selection and data extraction. Disagreements between the reviewers will be resolved by discussion until consensus is reached or after consultation with the research team when needed. Results will be reported with descriptive statistics and diagrammatic or tabular displayed information, accompanied by narrative summaries as explained in the JBI guidelines.

Ethics and dissemination Ethical approval for the conduct of this study is not required. We will analyse previously collected data for the proposed scoping review. Our results will be published in a peer-reviewed journal and disseminated through conferences and/or seminars.

\section{INTRODUCTION}

Advanced kidney disease is defined as an estimated glomerular filtration rate (eGFR) of less than $30 \mathrm{~mL} / \mathrm{min} / 1.73 \mathrm{~m}^{2}$, and marks a stage in the lives of patients during which they have to make treatment modality decisions as their disease progresses to end-stage

\section{Strength and limitations of this study}

The proposed scoping review will be the first paper to systematically search and map shared decisionmaking (SDM) interventions for treatment modality decisions in advanced kidney disease, evaluate the evidence on their reported outcomes and report on new developments or ongoing studies in this field.

- It will provide a comprehensive overview by collecting information from both quantitative and qualitative research, as well as the grey literature and key experts on SDM.

- Research in SDM is heterogeneous in its methodology and the reporting of outcomes; therefore a scoping review will be better suited to map, summarise and present this information than traditional systematic reviews or meta-analyses.

- Included studies will not undergo a formal quality assessment as scoping reviews attempt to provide an overview of all the existing evidence, regardless of its quality.

- Potentially relevant findings from papers written in other languages will be missed, as this study will only include papers written in English.

kidney disease (ESKD). As this process may take months or years, both patients and healthcare professionals face a considerable challenge in the anticipation of when kidney replacement therapy (KRT) will become necessary. Furthermore, questions regarding the eligibility of patients for all treatment options, the impact of these treatments on their lives, the concessions they are (not) willing to make and uncertainty regarding the outcomes they can expect, make this a difficult decision. Therefore, international guidelines in nephrology suggest shared decision-making (SDM) to help patients make timely treatment modality decisions that align with their preferences and values. ${ }^{1}$ Shared decision-making has been defined as a process during which patients, caregivers and healthcare professionals relate to and influence each other as they collaborate in making 
healthcare decisions. ${ }^{3}$ Patient decision aids (PtDAs) have been developed to support this decision-making process, and in recent years, healthcare outcomes, including patient-reported outcome measures (PROMs), have been defined for benchmarking, organisation of care and as novel tools to support the decision-making process. ${ }^{4-9}$

As the concept of SDM has been gaining traction in the medical community, the body of literature reporting on the involvement of patients in this decision-making process has been expanding accordingly. In response to this growing body of literature, efforts have been made to compile and summarize the available evidence on the subject. A systematic review on the barriers and facilitators for the implementation of SDM in clinical practice stated that gaps in the knowledge for the effective implementation of SDM in clinical practice remain and should be prioritized in future studies. ${ }^{10}$ Moreover, a systematic review on the implementation of PtDAs stated that the underlying issues that militate against their use, and more generally limit the adoption of SDM, are underspecified and underinvestigated. ${ }^{11}$ In addition, a series of Cochrane reviews concluded that there is high-quality evidence that PtDAs improve the knowledge of patients on their options and reduce decisional conflict, that the evidence for PtDAs in activating patients for decisionmaking and improving risk perceptions is moderate and that the evidence for PtDAs in improving congruence between decisions and personal values is growing. ${ }^{4}{ }^{12}$ Furthermore, when it comes to the effect of interventions to increase the use of SDM practices by healthcare professionals, another Cochrane review stated that it was uncertain whether any intervention is effective, because the certainty of the evidence is low or very low. ${ }^{3}$ Accordingly, a scoping review identified a number of interventions to promote the adoption of SDM in clinical practice, but due to heterogeneity in the assessments of their implementation and effectiveness, recommendations on the best strategies to promote the adoption of SDM could not be given. ${ }^{13}$ Finally, another scoping review identified multiple organisational-level and system-level characteristics that play a role in the implementation of SDM in routine care, and concluded that healthcare organisations should consider these characteristics if they wish to support the adoption of SDM. ${ }^{14}$ Of these reviews, only three report on the evidence for the effectiveness of SDM or PtDAs in the context of kidney disease, ${ }^{3414}$ and of the ten papers that are mentioned in these papers, only 4 were published. ${ }^{15-18}$ Therefore, the relevance of the statements made in these papers may be questioned for AKD or any other form of kidney disease.

When it comes to treatment modality decision-making in $\mathrm{AKD}$, no papers present a thorough overview of existing SDM interventions with evidence on any of their outcomes or novel developments in this field. Systematic reviews, including meta-analyses, have been written on: the perspectives of living with kidney failure ${ }^{19}$; factors influencing the decision-making process regarding treatment modalities for patients with $\mathrm{AKD}^{20-25}$; the readability of written materials for patients with chronic kidney disease $(\mathrm{CKD})^{2627}$; the effects of education and cognition of patients on $\mathrm{SDM}^{28-30}$; the validity of prognostic algorithms for this decision-making process ${ }^{31}$; advanced care planning ${ }^{32}{ }^{33}$ and treatment outcomes in the elderly. ${ }^{34-38}$ Furthermore, a preliminary search in the PubMed, Medline, Embase, Web of Science, Cochrane Library, Emcare, International Prospective Register of Systematic Reviews (PROSPERO), PsycINFO and Academic Search Premier databases did not identify any scoping reviews on this subject. Scoping reviews have been written on the clinical pathways for patients with CKD in the primary care setting and on factors influencing dialysis withdrawal. ${ }^{39} 40$ Additionally, a protocol for a scoping review on the information available for SDM with older patients with AKD considering their treatment options has been published. ${ }^{41}$ Finally, numerous narrative reviews and overview papers on these topics in the context of kidney failure have been published as well. ${ }^{42-63}$ All of these papers are either limited to a single aspect of the decision-making process or their methodological framework limits their validity due to uncertainties in the generalisability and reproducibility of the reported findings. This hampers adoption of the SDM concept by healthcare professionals and hinders further research and development on the subject.

Therefore, our aim is to write a comprehensive and up-to-date scoping review on SDM-interventions for treatment modality decisions in AKD. Our objectives are to map these SDM interventions to evaluate the evidence on their reported use and studied outcomes and to provide an overview of new interventions that are being developed or investigated. This will provide healthcare professionals and researchers with a much needed source of information on the subject and can reveal knowledge gaps facilitating further research and development. This article presents our study protocol.

\section{STUDY DEFINITIONS}

The following operational definitions will be used in this protocol:

- Advanced kidney disease: CKD-Kidney Disease Improving Global Outcomes (KDIGO) G4-G5A kidney failure. ${ }^{2}$

- Patients with AKD: all patients with $\mathrm{AKD} \geq 18$ years of age that have to make treatment modality decisions.

- Healthcare professionals: nephrologists, nurse practitioners, social workers and dietitians that are involved in the decision-making process regarding treatment modality choices.

- Treatment modality: kidney transplantation (living donor or deceased donor), haemodialysis (in-centre or home), peritoneal dialysis (ambulatory peritoneal dialysis or continuous automatic peritoneal dialysis) or conservative care management.

- PtDAs: tools designed to help people participate in decision-making about healthcare options, as defined 
by, but not limited to, the International Patient Decision Aid Standards collaboration. ${ }^{64}$

- SDM: the process in which patients, caregivers and healthcare professionals relate to and influence each other as they collaborate in making healthcare decisions. $^{3}$

- SDM intervention: any intervention in standard care supporting SDM between patients and healthcare professionals (eg, PtDAs, educational programme for patients or healthcare professionals, prognostic algorithms and peer support programme).

\section{STUDY AIM AND OBJECTIVES}

The proposed scoping review will systematically collect and synthesise information on the topic of SDM interventions for treatment modality decisions in AKD to:

- Provide a comprehensive and up-to-date overview for healthcare professionals.

- Explore and define knowledge gaps on the subject.

- Facilitate future research and development.

The objectives of the proposed scoping review are:

- To map all existing SDM interventions for treatment modality decisions in AKD.

- To evaluate the evidence of their reported use and studied outcomes.

- To provide an overview of interventions that are being developed or investigated.

\section{REVIEW QUESTIONS}

The questions and subsequent subquestions for the proposed scoping review are as follows:

1. What SDM interventions for treatment modality decisions in AKD have been developed?

- Which and how many treatment options are targeted by these interventions?

- What do these interventions consist of?

2. What is the evidence for the reported use and outcomes of these SDM interventions?

- Which of these interventions have been investigated for their outcomes?

- What are the reported effects of these interventions on the decision-making process, on the decisions made and on healthcare outcomes?

- How many of these interventions have been implemented in clinical practice as part of standard care?

3. What new SDM interventions are being developed or investigated?

- Are there any new SDM interventions for treatment modality decisions in AKD being created or studied?

- Will the creators report on the outcomes of these interventions?

- What outcomes will be reported?

- When can we expect the publication of these outcomes?

\section{METHODS AND ANALYSIS}

The proposed scoping review will be performed in accordance with the Joanna Briggs Institute's methodology for scoping reviews. ${ }^{65}$ Please refer to online supplementary appendix 1 for a flow chart depicting the study design. Two independent reviewers will participate in an iterative process of screening the literature, paper selection and data extraction on the basis of paper charting and data extraction tables. Please refer to online supplementary appendices 2 and 3 for draft versions of these tables. Disagreements between the reviewers will be resolved by discussion until a consensus is reached or after consultation with the research team when needed.

\section{Context and concept}

We will investigate the literature on SDM interventions for treatment modality decisions in the context of AKD, in both inpatient and outpatient care settings. To keep the focus of this review on interventions regarding treatment modality decisions, or interventions regarding a switch from treatment modalities, we will refrain from reviewing interventions that focus on advance care planning or the withdrawal from treatment. All developed tools will be investigated, whether they have been validated or not. When possible, we will report on outcomes of these SDM interventions as well. We expect that most papers will not report on outcomes, and that when they do, they will not compare these outcomes to standard care. Finally, we will provide an overview of SDM interventions under development or investigation and report on expected dates for the publication of their outcomes.

\section{Eligible study designs and papers}

The proposed scoping review will cover both qualitative and quantitative scientific literature, as well as the grey literature on SDM interventions for treatment modality decisions in AKD. Only literature written in English will be considered for inclusion. The following study designs and papers will be eligible for inclusion:

- Systematic reviews, meta-analyses, scoping reviews, overview papers, narrative reviews.

- Experimental and quasiexperimental study designs, that is, randomised or non-randomised controlled trials, controlled and uncontrolled pre-post studies and (multiple) interrupted time series.

- Quantitative descriptive and analytical observational studies, that is, retrospective and prospective cohort studies, case-control and cross-sectional studies, case series and case reports.

- Qualitative studies, using for example, grounded theory, phenomenology and study designs such as ethnography, action research and qualitative descriptions.

- Letters to the editors, professional opinion papers.

(International) guidelines, papers on the meetings of expert panels and available published research protocols of studies not yet completed.

\section{Databases and additional sources}

We will search the PubMed, Medline, Embase, Web of Science, Cochrane library, Emcare, PROSPERO, 
PsycINFO and Academic Search Premier databases for relevant, peer-reviewed, published papers and research protocols on the subject.

The search for grey literature and additional research protocols will include searches on electronic sources such as Open Grey, psycEXTRA, BIOSIS, researchgate.net, europepmc.org, clinicaltrials.gov, trialregister.net and Google Scholar.

The search for guidelines will include searches on the platforms of the KDIGO association, the Renal Physicians Association, the American Society of Nephrology, the Canadian Society of Nephrology, the National Institute for Health and Care Excellence, the European Renal association-European Dialysis and Transplant Association and the Kidney Health Australia-Caring for Australians with Renal Impairment Association.

Papers will be excluded from this review if they:

- Do not address SDM interventions for treatment modality decisions in AKD.

- Only address patients with an eGFR $>30 \mathrm{~mL} /$ $\min / 1,72 \mathrm{~m}^{2}$.

- Report on SDM for paediatric patients.

- Are written in any language other than English.

\section{Search strategy}

A three-step search strategy, as explained in the Joanna Briggs Institute Reviewer's Manual, will be followed. ${ }^{65}$ The first step, a limited search for peer-reviewed, published papers on the PubMed database, has already been performed. After this first step, a research librarian was consulted and an analysis of the words contained in the titles, abstracts and index terms generated the following list of keywords:

- Share, shared, sharing.

- Relation, relations.

- Decision, decisions, participation, empowerment.

- Medical, clinical.

- Treatment, making.

- Patient, nurse, physician, doctor.

- Advanced, chronic, end stage.

- Kidney, renal.

- Disease, diseases, failure.

- AKD, CKD, ESRD.

With the help of our research librarian, these keywords will subsequently be used for the second step in our search strategy, a secondary search across all included databases and sources. As grey literature resources often lack advanced search features, identifying relevant grey literature can be a time-consuming process and is often not reported transparently. To keep our search strategy manageable and reproducible, we will use search terms consistently between different resources and limit the screening process to a set number of pages, for example, the first 50 results. Additionally, we will report the resource name and URL, the dates searched and the used search terms. After this secondary search, the third step will be performed. We will examine the references of the identified papers that have been selected for full-text review and the papers that will be included in the proposed scoping review. The reviewers intend to contact the authors of papers for further information if this is deemed relevant. Additionally, the reviewers intend to contact experts on SDM, identified through the literature, by phone or by email to inquire on new SDM interventions that are being developed or on ongoing studies in this field. Each search query and additional steps relating to the search of the proposed scoping review will be published as appendices in the scoping review.

\section{Study selection}

After the removal of duplicates, the results of the secondary search will be imported in RefWorks V.2.0. Both reviewers will independently screen all titles and abstracts and select papers they deem eligible for inclusion. After this process, both reviewers will compare their results and decide which papers to include. Finally, both reviewers will screen and select references from all included papers and repeat the same process for this selection.

\section{Data extraction}

The data of interest will be extracted with the data extraction tables by the two reviewers and entered into spreadsheets in Microsoft Excel V.16. If the data in a single paper is relevant to multiple research questions, these data will then be extracted using multiple tables. Results will be categorised according to the review questions and charted in an iterative process, allowing the reviewers to continuously update these charts when additional unforeseen data are encountered.

\section{PRESENTATION OF THE RESULTS}

All extracted data will be presented in tabular or diagrammatic form. First, a table with the details of all included papers will be given. After this, the results will be presented in the following main conceptual categories that are based on the research questions that form the basis of this scoping review:

- Number and characteristics of SDM interventions.

- Basic demographics of patients and outcome variables used in the included papers.

- Reported effects of the SDM interventions on treatment modality decisions that have been made, and if applicable, reported differences with comparators.

- Reported effects of the SDM interventions on the decision-making process, and if applicable, reported differences with comparators.

- Reported effects of the SDM interventions on healthcare outcome measures, and if applicable, reported differences with comparators.

- Overlapping themes in the reported outcomes.

- The validation of SDM interventions.

- Knowledge gaps on the subject.

- Implementation in daily practice.

- New and/or ongoing developments and/or studies on SDM interventions. 
Descriptive statistics will be used to provide an overview of the basic demographics and outcome variables of the included papers. Continuous data will be expressed as a mean $\pm \mathrm{SD}$ or as the median (IQR) where appropriate. Categorical data will be expressed as frequencies (\%), unless otherwise stated. IBM SPSS Statistics V.23 will be used for all statistical analyses. Narrative summaries will accompany the tabulated and/or diagrammatic results and describe how the results relate to the research questions regarding SDM interventions for treatment modality decisions in AKD.

Qualitative data will be displayed in tabular or diagrammatic form. A combination of inductive and deductive approaches will be used to analyse the data (eg, open coding or the framework approach) in Atlast.ti. Emergent themes will be discussed in the research team. It is expected that the identification of SDM interventions and their reported effects will further refine the conceptual categories for data presentation.

\section{Patient and public involvement}

There was no patient or public involvement in the design of this scoping review protocol.

\section{ETHICS AND DISSEMINATION}

Ethical approval for the conduct of this study is not required because this scoping review will analyse previously collected data. Results will be published in a peerreviewed journal and disseminated through conferences and/or seminars.

\section{CONCLUSION}

International guidelines suggest SDM to support patients with AKD make treatment modality decisions as their disease progresses towards ESKD. However, papers that present a thorough overview of SDM interventions for these decisions, evidence on any of their outcomes or new interventions that are being developed or investigated are lacking. This leaves healthcare professionals and researchers guessing, which hampers further implementation, research and development. Therefore, the proposed scoping review will map these SDM interventions for treatment modality decisions in $\mathrm{AKD}$, summarise and report on the effectiveness of these interventions and report on new developments or ongoing studies in this field. Our objectives are to provide a comprehensive and up-to-date overview for healthcare professionals and researchers, explore and define knowledge gaps and facilitate future research and development.

\section{Twitter Anne M Stiggelbout @AMStiggelbout}

Acknowledgements The authors thank the research librarian Dr JW Schoones of the Leiden University Medical Center for his help in defining and generating the keywords necessary to perform the subsequent search query.

Contributors NE is the primary and corresponding author and was responsible for the first and all subsequent drafts of this scoping review protocol. GeG, MvdD, PvdN, WJB and AMS all participated in discussions on the study design. Additionally, they contributed to the design of this study protocol and revised drafts critically for improvements. All six authors approved the final version to be published. All authors have agreed to be held accountable for all aspects of this study protocol.

Funding This work was supported by ZonMW as part of the 'Experiment Uitkomst indicatoren Santeon'.

Disclaimer None declared.

Competing interests None declared.

Patient consent for publication Not required.

Provenance and peer review Not commissioned; externally peer reviewed.

Open access This is an open access article distributed in accordance with the Creative Commons Attribution Non Commercial (CC BY-NC 4.0) license, which permits others to distribute, remix, adapt, build upon this work non-commercially, and license their derivative works on different terms, provided the original work is properly cited, appropriate credit is given, any changes made indicated, and the use is non-commercial. See: http://creativecommons.org/licenses/by-nc/4.0/.

\section{ORCID iDs}

Noel Engels http://orcid.org/0000-0002-5101-554X

Anne M Stiggelbout http://orcid.org/0000-0002-6293-4509

\section{REFERENCES}

1 Renal Physicians Association. Shared decision making in the appropriate initiation of and withdrawal from dialysis. 2nd edn. Rockville, MD: Renal Physicians Association, 2010.

2 KDIGO. 2012 clinical practice guideline for the evaluation and management of chronic kidney disease, 2013. Available: http://www. kidney-international.org

3 Légaré F, Adekpedjou R, Stacey D, et al. Interventions for increasing the use of shared decision making by healthcare professionals. Cochrane Database Syst Rev 2018;7:CD006732.

4 Stacey D, Légaré F, Lewis K, et al. Decision AIDS for people facing health treatment or screening decisions. Cochrane Database Syst Rev 2017;4:CD001431.

5 Verberne WR, Das-Gupta Z, Allegretti AS, et al. Development of an international standard set of value-based outcome measures for patients with chronic kidney disease: a report of the International Consortium for health outcomes measurement (ICHOM) CKD Working group. Am J Kidney Dis 2019;73:372-84.

6 Viecelli AK, Tong A, O'Lone E, et al. Report of the Standardized Outcomes in Nephrology-Hemodialysis (SONG-HD) Consensus Workshop on Establishing a Core Outcome Measure for Hemodialysis Vascular Access. Am J Kidney Dis 2018;71:690-700.

7 Tong A, Sautenet B, Poggio ED, et al. Establishing a core outcome measure for graft health: a standardized outcomes in NephrologyKidney transplantation (SONG-Tx) consensus workshop report. Transplantation 2018;102:1358-1366.

8 Manera KE, Tong A, Craig JC, et al. Standardized outcomes in Nephrology-Peritoneal dialysis (SONG-PD): study protocol for establishing a core outcome set in PD. Perit Dial Int 2017;37:639-47.

9 Breckenridge K, Bekker HL, Gibbons E, et al. How to routinely collect data on patient-reported outcome and experience measures in renal registries in Europe: an expert consensus meeting. Nephrol Dial Transplant 2015;30:1605-14.

10 Légaré F, Ratté S, Gravel K, et al. Barriers and facilitators to implementing shared decision-making in clinical practice: update of a systematic review of health professionals' perceptions. Patient Educ Couns 2008;73:526-35.

11 Elwyn G, Scholl I, Tietbohl C, et al. "Many miles to go ...": a systematic review of the implementation of patient decision support interventions into routine clinical practice. BMC Med Inform Decis Mak 2013;13.

12 Stacey D, Légaré F, Col NF, et al. Decision AIDS for people facing health treatment or screening decisions. Cochrane Database Syst. Rev 2014;28.

13 Siyam T, Shahid A, Perram M, et al. A scoping review of interventions to promote the adoption of shared decision-making (SDM) among health care professionals in clinical practice. Patient Educ Couns 2019;102:1057-66.

14 Scholl I, LaRussa A, Hahlweg P, et al. Organizational- and systemlevel characteristics that influence implementation of shared decision-making and strategies to address them - a scoping review. Implement Sci 2018;13:40.

15 Manns BJ, Taub K, Vanderstraeten C, et al. The impact of education on chronic kidney disease patients' plans to initiate dialysis with selfcare dialysis: a randomized trial. Kidney Int 2005;68:1777-83. 
16 Registered Nurses' Association of Ontario. Decision support for adults living with chronic kidney disease, 2009. Available: http:// rnao.ca/bpg/guidelines/decision-support-adults-livingchronickidney-disease

17 Fortnum D, Smolonogov T, Walker R, et al. 'My kidneys, my choice, decision aid': supporting shared decision making. J Ren Care 2015;41:81-7.

18 Mollicone D, Pulliam J, Lacson E. The culture of education in a large dialysis organization: informing patient-centered decision making on treatment options for renal replacement therapy. Semin Dial 2013;26:143-7.

19 Bailey PK, Hamilton AJ, Clissold RL, et al. Young adults' perspectives on living with kidney failure: a systematic review and thematic synthesis of qualitative studies. BMJ Open 2018;8:e019926.

20 Murray MA, Brunier G, Chung JO, et al. A systematic review of factors influencing decision-making in adults living with chronic kidney disease. Patient Educ Couns 2009;76:149-58.

21 Morton RL, Tong A, Howard K, et al. The views of patients and carers in treatment decision making for chronic kidney disease: systematic review and thematic synthesis of qualitative studies. BMJ 2010;340:c112.

22 Harwood L, Clark AM. Understanding pre-dialysis modality decisionmaking: a meta-synthesis of qualitative studies. Int J Nurs Stud 2013;50:109-20.

23 Tong A, Hanson CS, Chapman JR, et al. The preferences and perspectives of nephrologists on patients' access to kidney transplantation: a systematic review. Transplantation 2014;98:682-91.

24 Hussain JA, Flemming K, Murtagh FE, et al. Patient and health care professional decision-making to commence and withdraw from renal dialysis: a systematic review of qualitative research. Clin J Am Soc Nephrol 2015;10:1201-15

25 Winterbottom A, Bekker HL, Conner M, et al. Choosing dialysis modality: decision making in a chronic illness context. Health Expect 2014:17:710-23.

26 Morony S, Flynn M, McCaffery KJ, et al. Readability of written materials for CKD patients: a systematic review. Am J Kidney Dis 2015;65:842-50.

27 Winterbottom A, Conner M, Mooney A, et al. Evaluating the quality of patient leaflets about renal replacement therapy across UK renal units. Nephrol Dial Transplant 2007;22:2291-6.

28 Devoe DJ, Wong B, James MT, et al. Patient education and peritoneal dialysis modality selection: a systematic review and metaanalysis. Am J Kidney Dis 2016;68:422-33.

29 Berger I, Wu S, Masson P, et al. Cognition in chronic kidney disease: a systematic review and meta-analysis. BMC Med 2016;14:206.

30 Taylor DM, Fraser SDS, Bradley JA, et al. A systematic review of the prevalence and associations of limited health literacy in CKD. Clin J Am Soc Nephrol 2017:12:1070-84.

31 Ramspek CL, Voskamp PW, van Ittersum FJ, et al. Prediction models for the mortality risk in chronic dialysis patients: a systematic review and independent external validation study. Clin Epidemiol 2017;9:451-64

32 Lim CED, Ng RWC, Cheng NCL, et al. Advance care planning for haemodialysis patients. Cochrane Database Syst Rev 2016;7:CD010737.

33 O'Halloran P, Noble H, Norwood K, et al. Advance care planning with patients who have end-stage kidney disease: a systematic realist review. J Pain Symptom Manage 2018;56:795-807.

34 Foote C, Kotwal S, Gallagher M, et al. Survival outcomes of supportive care versus dialysis therapies for elderly patients with end-stage kidney disease: a systematic review and meta-analysis. Nephrology 2016;21:241-53.

35 Wongrakpanich S, Susantitaphong $\mathrm{P}$, Isaranuwatchai $\mathrm{S}$, et al. Dialysis therapy and conservative management of advanced chronic kidney disease in the elderly: a systematic review. Nephron 2017; $137: 178-89$

36 Verberne WR, Geers ABMT, Jellema WT, et al. Comparative survival among older adults with advanced kidney disease managed conservatively versus with dialysis. Clin J Am Soc Nephrol 2016;11:633-40.

37 Verberne WR, Dijkers J, Kelder JC, et al. Value-Based evaluation of dialysis versus conservative care in older patients with advanced chronic kidney disease: a cohort study. BMC Nephrol 2018;19:205.

38 Brown L, Gardner G, Bonner A. A comparison of treatment options for management of end stage kidney disease in elderly patients: a systematic review protocol. JBI Database Syst Rev Implement Rep.

39 Elliott MJ, Gil S, Hemmelgarn BR, et al. A scoping review of adult chronic kidney disease clinical pathways for primary care. Nephrol Dial Transplant 2017;32:838-46.
40 Qazi HA, Chen H, Zhu M. Factors influencing dialysis withdrawal: a scoping review. BMC Nephrol 2018:19:96.

41 Raj R, Ahuja KDK, Frandsen M, et al. Older patient considering treatment for advanced renal disease: protocol for a scoping review of the information available for shared decision-making. BMJ Open 2016;6:e013755

42 Stryckers M, Nagler EV, Van Biesen W. The need for accurate risk prediction models for road mapping, shared decision making and care planning for the elderly with advanced chronic kidney disease. Pril 2016;37:33-42

43 Couchoud C, Hemmelgarn B, Kotanko P, et al. Supportive care: time to change our prognostic tools and their use in CKD. Clin J Am Soc Nephrol 2016;11:1892-901.

44 Kadatz MJ, Lee ES, Levin A. Predicting progression in CKD: perspectives and precautions. Am J Kidney Dis 2016;67:779-86.

45 Murtagh FEM, Burns A, Moranne O, et al. Supportive care: comprehensive conservative care in end-stage kidney disease. Clin $J$ Am Soc Nephrol 2016;11:1909-14.

46 Murray MA, Bissonnette J, Kryworuchko J, et al. Whose choice is it? shared decision making in nephrology care. Semin Dial 2013;26:169-74.

47 Llewellyn S. Concept clarification: uncertainty in individuals with chronic kidney disease. Nephrol Nurs J 2017;44:513-39.

48 Cassidy BP, Getchell LE, Harwood L, et al. Barriers to education and shared decision making in the chronic kidney disease population: a narrative review. Can J Kidney Health Dis 2018;5:205435811880332.

49 Wilson S, Dhar A, Tregaskis P, et al. Known unknowns: examining the burden of neurocognitive impairment in the end-stage renal failure population. Nephrology 2018;23:501-6.

50 Santos J, Fonseca I. Incorporating scoring risk models for care planning of the elderly with chronic kidney disease. Curr Gerontol Geriatr Res 2017;2017:8067094:1-6.

51 Rosansky SJ, Schell J, Shega J, et al. Treatment decisions for older adults with advanced chronic kidney disease. BMC Nephrol 2017;18:200.

52 Morton RL, Kurella Tamura M, Coast J, et al. Supportive care: economic considerations in advanced kidney disease. Clin J Am Soc Nephrol 2016;11:1915-20.

53 Schmidt RJ. Advance care planning for patients approaching endstage kidney disease. Semin Nephrol 2017;37:173-80.

54 Green JA, Boulware LE. Patient education and support during CKD transitions: when the possible becomes probable. Adv Chronic Kidney Dis 2016;23:231-9.

55 Collister D, Russell R, Verdon J, et al. Perspectives on optimizing care of patients in multidisciplinary chronic kidney disease clinics. Can J Kidney Health Dis 2016;3:122.

56 Berger JR, Jaikaransingh V, Hedayati SS. End-Stage kidney disease in the elderly: approach to dialysis initiation, choosing modality, and predicting outcomes. Adv Chronic Kidney Dis 2016;23:36-43.

57 MacPhail A, Ibrahim JE, Fetherstonhaugh D, et al. The overuse, underuse, and misuse of dialysis in ESKD patients with dementia. Semin Dial 2015;28:490-6.

58 Ghahramani N. Potential impact of peer mentoring on treatment choice in patients with chronic kidney disease: a review. Arch Iran Med 2015;18:239-43.

59 Muthalagappan S, Johansson L, Kong WM, et al. Dialysis or conservative care for frail older patients: ethics of shared decisionmaking. Nephrol Dial Transplant 2013;28:2717-22.

60 Brown EA, Johansson L. Dialysis options for end-stage renal disease in older people. Nephron Clin Pract 2011;119 Suppl 1:c10-13.

61 Campbell KH, Dale W, Stankus N, et al. Older adults and chronic kidney disease decision making by primary care physicians: a scholarly review and research agenda. $J$ Gen Intern Med 2008;23:329-36

62 White Y, Fitzpatrick G. Dialysis: prolonging life or prolonging dying? ethical, legal and professional considerations for end of life decision making. Edtna Erca J 2006;32:99-103.

63 Pfettscher SA. Making decisions about end-stage renal disease treatment: a review of theories and issues. Adv Ren Replace Ther 1997:4:81-8.

64 International Patient Decision Aid Standards(IPDAS). Available: http:// ipdas.ohri.ca/what.html

65 Peters MDJ, Godfrey C, Mclnerney P. Chapter 11: Scoping reviews. In: Aromataris E, Munn Z, eds. Joanna Briggs Institute reviewer's manual. The Joanna Briggs Institute, 2017. https://reviewersmanual. joannabriggs.org/ 